\title{
Noise-Induced Signal Enhancement in Heterogeneous Neural Networks
}

\author{
Michael J. Barber and Babette K. Dellen \\ Institut für Theoretische Physik, Universität zu Köln, D-50937 Köln, Germany \\ mjb@thp.uni-koeln.de bd@thp.uni-koeln.de
}

\begin{abstract}
Neural networks can represent complex functions, but are often constructed of very simple units. We investigate the limitations imposed by such a simple unit, the McCulloch-Pitts neuron. We explore the role of stochastic resonance in units of finite precision and show how to construct neural networks that overcome the limitations of single units.
\end{abstract}

\section{Introduction}

Neural networks are often constructed of very simple model neurons. Typically, model neurons have a particular threshold or bias, and saturate to a fixed value for either strong or weak inputs, yielding a so-called sigmoidal activation or "squashing" function. While individual units are simple, it is well known that neural networks can represent complex functions. In this work, we will investigate the degree to which the simple dynamics of an individual unit limit the inputs that can be represented, and how these limitations can be overcome.

To do this, we consider the response of model neurons to a variety of signals. The model neurons have limited precision, which we implement by including noise in the systems. We consider noise that is intrinsic to the neurons, and thus has identical statistics for each of the units in the neural networks.

Noise is usually viewed as limiting the sensitivity of a system, but nonlinear systems can show an optimal response to weak or subthreshold signals when a non-zero level of noise is added to the system. This phenomenon is called stochastic resonance (SR) [2]. SR is seen in many systems, ranging from resonant cavities to neural networks to the onset of ice ages [5]. For example, a noise-free, subthreshold neuronal input can occasionally become suprathreshold when noise is added, allowing some character of the input signal to be detected.

Collins et al.[1] showed, in a summing network of identical Fitzhugh-Nagumo model neurons, that an emergent property of SR in multi-component systems is that the enhancement of the response becomes independent of the exact value of the noise variance. This allows networks of elements with finite precision to take advantage of SR for diverse inputs. To build upon the findings of Collins et al., we consider networks of simpler model neurons, but these are allowed to have different dynamics. In particular, we examine noisy McCulloch-Pitts (McP)

V.N. Alexandrov et al. (Eds.): ICCS 2001, LNCS 2074, pp. 996-999, 2001.

(C) Springer-Verlag Berlin Heidelberg 2001 
neurons [3] with a distribution of thresholds. We construct heterogeneous networks that perform better than a homogeneous network with the same number of noisy McP neurons and similar network architecture.

\section{Results}

To investigate the effect of noise on signal transduction in networks of thresholding units, we constructed a network of noisy McCulloch-Pitts neurons. A McP neuron is a simple thresholding unit, which can be expressed mathematically as a step function. When the total input to a neuron (signal plus noise) exceeds its threshold, the neuron activates, firing an action potential or "spike." The presence of a non-zero level of noise can make the neuron more sensitive to weak signals; for example, a subthreshold signal can occasionally be driven above the neuronal threshold by the noise. The intrinsic neuronal noise is modeled in this work as independent, identically distributed Gaussian white noise that is added to the input signal. We use the standard deviation of the Gaussian as a measure of the noise strength.

The network architecture is very simple: an input layer of $N$ noisy $\mathrm{McP}$ neurons is connected to a single linear output neuron. Each synaptic weight is of identical strength, so the output neuron calculates the mean value of the input neuron activation states. Each input unit is presented the same analog signal, but with a different realization of the intrinsic neuronal noise. The uncorrelated noises increase only by a factor of $\sqrt{N}$ on average across the population of neurons, while the coherent input signal is strengthened by a factor of $N$. This yields an increased signal-to-noise ratio and a more sensitive neural network.

We reconstruct the input signal by convolving the output neuron response with a linear filter [4]. The filter is generated to minimize a quadratic difference between the reconstructed signal and the input signal; the resulting filter for $\mathrm{McP}$ neurons is a low-pass filter. We quantitatively compare the reconstruction with the original signal, and make use of the standard correlation coefficient $r^{2}$ as a direct measure of the similarity between signal and reconstruction.

A single McP neuron shows a characteristic stochastic resonance profile in response to weak signals (generated here as a random walk with zero mean and a typical variance of one) with different noise intensities (Fig. 1a, green circles). As the noise increases, there is an initially enhanced network response, which then drops off as the noise further increases, overwhelming the signal. However, networks of McP neurons, all of which have identical thresholds, have a broad plateau of noise-enhanced responses (Fig. 1a, blue squares and red triangles), as well as an overall enhanced response to a signal. This is similar to the findings of Collins et al.[1].

The behavior seen in Fig. 1a appears to indicate that noise is a good thing to have in neurons, but it was calculated under the assumption of weak signals. To expand upon this assumption, we consider the ability of networks of McP neurons to reconstruct signals of different strength. In this instance, "strength" can be understood as the difference between the mean value of the input signal 

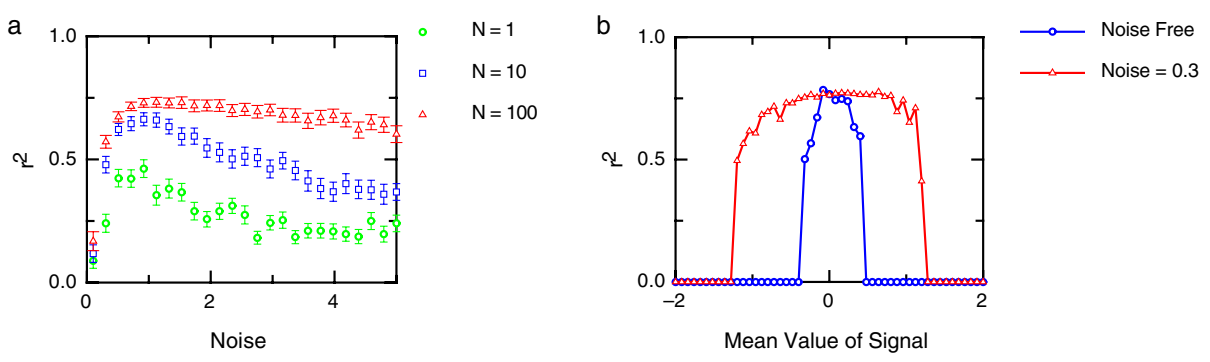

Fig. 1. Stochastic resonance is seen in networks of McCulloch-Pitts neurons. (a) With an increasing number $N$ of neurons, the stochastic resonance peak broadens, losing the strong dependence on a particular noise level. The network responses shown are the average result for 50 zero-mean, random walk inputs; each signal is weak and normally subthreshold, with the neuronal thresholds equal to one. (b) The presence of noise widens the range over which signals are accurately detected and reconstructed. Here, we show a network of 100 neurons with thresholds of zero.

and the value of the neuronal thresholds, so we vary the signal mean while keeping the thresholds and the noise intensity fixed. In this manner, we see that the presence of noise broadens the range of signal strengths that the network can detect (Fig. 1b). This is the mirror image of the weak-signal stochastic resonance effect: strong input signals may be supra-threshold at all times, so that the McP neuron fires at each sampling time, but the presence of noise can drive the total signal below the threshold and improve the network sensitivity.

The network sensitivity increases as the number of input neurons increases (Fig. 2a). For large numbers of neurons, adding more units widens the range of signal detection, but does not significantly improve the peak reconstruction performance of the network.

The networks shown in Fig. 2a indicate that there is only a minimal widening of the range of signal detection for a large increase in the number of neurons. However, these networks were homogeneous, with each neuron having an identical threshold. A natural extension is to consider heterogeneous systems, where the thresholds are not uniform. We divide the neurons evenly into two subpopulations of different thresholds. The neurons in each subpopulation are identical, and the subpopulations have identical noise intensities, differing only in the thresholds. Again applying the signal reconstruction procedure, we see that the signal detection range can be more efficiently widened in this way than with a single homogeneous population of neurons (Fig. 2b). The use of multiple thresholds increases the range of signal detection without any significant loss in the quality of signal detection.

\section{Conclusion}

We have constructed a network of heterogeneous McP neurons that outperforms similar networks of homogeneous McP neurons. The network architectures are 

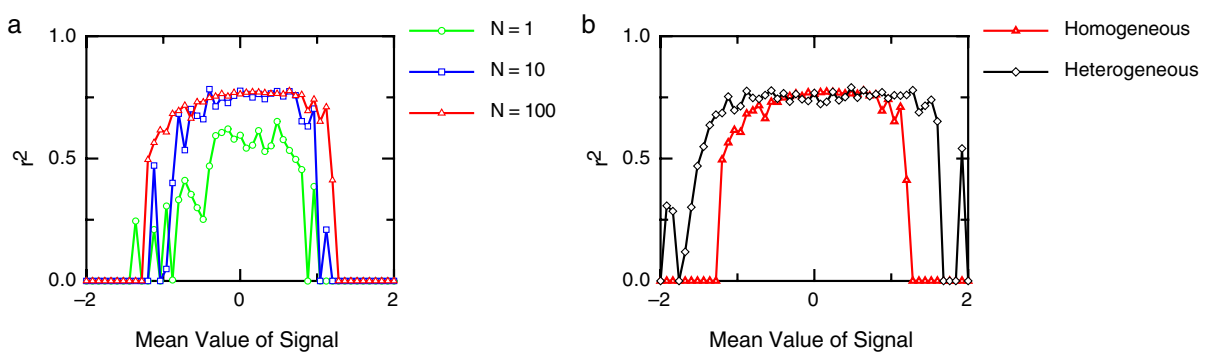

Fig. 2. Comparison of homogeneous and heterogeneous networks. (a) The range of effective signal detection and reconstruction increases gradually as the number of neurons increases. Every neuron in each of the networks shown here has an identical threshold, equal to zero. (b) Segregating the neurons into two groups with different thresholds leads to a greater range of effective signal detection and reconstruction than an increase in the number of neurons with uniform thresholds. The heterogeneous network has 10 neurons with a threshold of -0.65 and 10 neurons with a threshold of +0.65 . The homogeneous network is identical to the 100 neuron network shown in (a).

identical, with the only difference being the distribution of neuronal thresholds. The heterogeneous system is sensitive to a wider range of signals than the homogeneous systems. Such networks of heterogeneous units are easily implemented, and could serve as simple models of many diverse natural and artificial systems.

A network of low-precision neurons (or other units) can take advantage of stochastic resonance to accurately encode and transmit an input. The collective properties of these systems can exceed the limitations of a single element. A careful consideration of properties of the elements, such as their thresholds, may yield even further improvements in the performance of the system.

\section{Acknowledgements}

We thank John Clark, Manfred Ristig, and Jürgen Hescheler for valuable discussion. This work was supported in part by Graduiertenkolleg Azentrische Kristalle GK549 of the DFG.

\section{References}

[1] J.J. Collins, C.C. Chow, and T.T. Imhoff. Stochastic resonance without tuning. Nature, 376:236-238, July 1995.

[2] L. Gammaitoni, P. Hänggi, P. Jung, and F. Marchesoni. Stochastic resonance. Rev. Mod. Phys., 70(1):223-87, January 1998.

[3] J. Hertz, A. Krogh, and R.G. Palmer. Introduction to the Theory of Neural Computation. Addison-Wesley Publishing Company, Reading, MA, 1991.

[4] F. Rieke, D. Warland, R.R. de Ruyter van Steveninck, and W. Bialek. Spikes: Exploring the Neural Code. MIT Press, Cambridge, MA, 1997.

[5] K. Wiesenfeld and F. Moss. Stochastic resonance and the benefits of noise: From ice ages to crayfish and SQUIDs. Nature, 373:33-36, January 1995. 